\title{
Efeito da fitase na biodisponibilidade do fósforo do farelo de arroz em frangos de corte ${ }^{(1)}$
}

\author{
Ademir José Conte ${ }^{(2)}$, Antonio Soares Teixeira( ${ }^{(3)}$, Agostinho Valente de Figueirêdo(4), \\ Dorinha Miriam Silber Schimidt Vitti( ${ }^{(5)}$ e José Cleto da Silva Filho(3)
}

\begin{abstract}
Resumo - O trabalho objetivou determinar a ação da enzima fitase sobre a biodisponibilidade do fósforo do farelo de arroz em dietas de frangos de corte, pela técnica de diluição isotópica. Os tratamentos consistiram de uma dieta sem farelo de arroz integral e sem suplementação de fósforo inorgânico $(0,388 \%$ de $P$ total $)$, e quatro dietas com $15 \%$ de farelo de arroz integral, sem suplementação de fósforo inorgânico ( $(0,56 \%$ de P total) e com quatro níveis de fitase $(0,400,800$ e 1,200 FTU $/ \mathrm{kg})$. No quinto dia do experimento, cada ave recebeu, via peritonial, $3,5 \mathrm{MBq} d{ }^{32} \mathrm{P}$. Nos quatro dias subseqüentes, coletaram-se amostras de sangue e excretas para as análises. As perdas endógenas fecais, a absorção aparente e verdadeira de fósforo e o fósforo no plasma aumentaram de maneira significativa $(\mathrm{P}<0,01)$ com a utilização de níveis crescentes de fitase. A biodisponibilidade do fósforo foi de $38,06 \%$ na dieta com farelo de arroz e sem enzima, e aumentou para 51,54\%, 61,31\% e 59,54\% com a utilização de 400, 800 e $1.200 \mathrm{FTU} / \mathrm{kg}$, respectivamente. Por meio de regressão quadrática, determinou-se a máxima biodisponibilidade de P, que foi de $60,8 \%$ com a utilização de $982 \mathrm{FTU} / \mathrm{kg}$. A biodisponibilidade do fósforo do farelo de arroz, determinada por fracionamento, foi de $28,1 \%$ e aumentou de forma linear $(\mathrm{P}<0,05)$ para 38,3\%, 50,2\% e 54,0\%, com a utilização de 400, 800 e 1.200 FTU/kg.
\end{abstract}

Termos para indexação: absorção, excreção, nutrientes, isótopos, técnicas de rastreio.

\section{Effect of phytase on the bioavailability of phosphorus of rice bran in broilers chickens}

\begin{abstract}
This work has intended to determine the action of phytase on the bioavailability of phosphorus of rice bran in the diet for broiler chickens through the isotopic dilution technique. The treatments consisted of a diet based on corn and soybean meal, without supplementation of inorganic phosphorus $(0.388 \%$ of total $\mathrm{P})$ and four diets of $15 \%$ of whole rice bran without supplementation of inorganic phosphorus $(0.56 \%$ of total $\mathrm{P})$ with four levels of phytase $(0,400,800$ and $1,200 \mathrm{FTU} / \mathrm{kg})$. On the fifth day of the experiment, each bird was given, peritoneal via, $3.5 \mathrm{MBq}$ of ${ }^{32} \mathrm{P}$. On the four subsequent days, blood and excreta samples were collected for further analyses. The fecal endogenous losses, plasma apparent and true absorption of phosphorus increased in a significant manner $(\mathrm{P}<0.01)$ with the use of growing levels of phytase in the diet. The bioavailability of phosphorus was of $38.06 \%$ in the diet with rice bran and without enzyme and increased to $51.54 \%, 61.31 \%$ and $59.54 \%$ using 400,800 and $1,200 \mathrm{FTU} / \mathrm{kg}$, respectively. Through quadratic regression the point of maximum bioavailability of $60.8 \%$ was determined with the use of $982 \mathrm{FTU} / \mathrm{kg}$. Phosphorus bioavailability of rice bran, determined by splitting, was of $28.1 \%$ and raised in a linear form $(\mathrm{P}<0.05)$ to $38.3 \%, 50.2 \%$ and $54 \%$ with the use of 400,800 and $1,200 \mathrm{FTU} / \mathrm{kg}$.
\end{abstract}

Index terms: absorption, excretion, nutrients, isotopes, tracer techniques.

(1) Aceito para publicação em 31 de maio de 2001 . Extraído da tese de doutorado apresentada pelo primeiro autor à Universidade Federal de Lavras (Ufla), Lavras, MG

(2) Escola Agrotécnica Federal de Cuiabá, BR 364, km 329 , CEP 78106-000 Cuiabá, MT. E-mail: ajconte@zaz.com.br (3) Ufla, Dep. de Zootecnia, Caixa Postal 37, CEP 37200-000 Lavras, MG. E-mail: asoarest@ufla.br, cleto@ufla.br

(4) Universidade Federal do Piauí, Centro de Ciências Agrárias, Campus de Socopo, CEP 64049-550 Teresina, PI

(5) Universidade de São Paulo, Centro de Energia Nuclear na Agricultura, Caixa Postal 32, CEP 13400-970 Piracicaba, SP. E-mail: dovitti@cena.usp.br
Introdução

Muitos estudos têm sido realizados para determinar as exigências nutricionais das aves, visando obter uma alimentação que proporcione o máximo desempenho e que tenha menor custo. Dentre os minerais, destaca-se o fósforo $(\mathrm{P})$, pela sua participação em inúmeras funções do organismo animal e pelo seu elevado custo na suplementação nas rações das 
aves. Nos alimentos de origem vegetal, que constituem mais de $90 \%$ das dietas de frangos, o P está em grande parte complexado e indisponível.

A utilização do $\mathrm{P}$ dos alimentos de origem vegetal pelos animais monogástricos está diretamente relacionada com o conteúdo de fitato, já que o P não é utilizado por esses animais, devido à ausência da enzima fitase no seu trato digestivo. A fitase atua hidrolisando o fitato, liberando o $\mathrm{P}$ e permitindo sua assimilação pelo animal. Normalmente, considera-se que apenas $30 \%$ do $\mathrm{P}$ dos vegetais seja disponível para animais monogástricos (National Research Council,1994; Rostagno et al.,1994). Entretanto, a quantidade de fitato é muito variável entre as espécies vegetais, afetando diretamente a biodisponibilidade do P. Assim, segundo Borges (1997), a biodisponibilidade do $\mathrm{P}$ do milho é de $33 \%$, do farelo de soja de $42 \%$ e do farelo de arroz de apenas $14 \%$.

$\mathrm{O}$ farelo de arroz é, entre os alimentos de origem vegetal, o que possui uma das maiores porcentagens de $\mathrm{P}$ total $(1,5 \%)$, enquanto o milho possui $0,28 \%$ e o farelo de soja, $0,65 \%$ (National Research Council,1994). Por outro lado, é o alimento que possui uma das menores taxas de disponibilidade de $\mathrm{P}$, devido ao seu alto teor de fitato. Segundo Nelson (1967), o farelo de arroz tem de 5,1\% a 8,6\% de fitato na sua composição, embora a maioria dos demais cereais possuam entre $1 \%$ e $2 \%$ de fitato.

$\mathrm{O}$ fato de que a produção de fitase endógena em aves é quase nula e o $\mathrm{P}$ complexado na forma de fitato torna-o indisponível originou estudos para utilização da enzima fitase exógena em rações para aves.

Muitos fungos, bactérias e leveduras produzem a enzima fitase. Nelson et al. (1968) foram os primeiros a utilizar a enzima fitase produzida por cultivos de Aspergillun ficuum em dietas para frangos, obtendo resultados benéficos. Apesar disso, somente no final da década de 1980, a produção de fitase atingiu escala comercial. Atualmente a fitase é produzida por diversas empresas do ramo, a partir de fungos do gênero Aspergillus, por meio de técnicas de recombinação do DNA. Segundo Borges (1997), a fitase aumenta a biodisponibilidade do $\mathrm{P}$ em cerca de $50 \%$, e reduz a sua excreção fecal e urinária em proporções semelhantes.

O conhecimento da biodisponibilidade e da eficiência de absorção do $\mathrm{P}$ da dieta pelo animais e os fatores que a regulam permitem transformar a exigência líquida em exigência dietética diária. Dessa forma, valores de biodisponibilidade e de eficiência de absorção super ou subestimados podem levar a um fornecimento inadequado do elemento para determinada categoria animal, o que pode acarretar prejuízos irreparáveis no processo de crescimento do animal, além de elevados prejuízos financeiros e ambientais quando a biodisponibilidade é subestimada.

Existem vários métodos para determinar a biodisponibilidade de nutrientes nos alimentos: o "slope ratio", o método das abscissas e a técnica de diluição isotópica. Segundo Figueirêdo (1998), a técnica de diluição isotópica é a única que torna possível a determinação das perdas endógenas do elemento. De acordo com Bellaver et al. (1983), técnicas como a "slope ratio" levam em conta apenas a absorção aparente do elemento e, por isso, subestimam o seu valor real. Assim, a biodisponibilidade obtida pela técnica de radioisótopos é considerada real, enquanto as demais são aparentes, pois não determinam as perdas endógenas do elemento. Vitti et al. (1991) citam que um dos primeiros trabalhos realizados para determinar a digestibilidade verdadeira do $\mathrm{P}$ em forragens foi realizado por Kleiber et al. (1951) que obtiveram valores de $50 \%$, ao passo que a digestibilidade aparente foi de $12 \%$, o que ilustra a enorme diferença que pode haver entre a disponibilidade aparente e verdadeira de um elemento.

O objetivo deste trabalho foi determinar o efeito da enzima fitase sobre a biodisponibilidade do $\mathrm{P}$ do farelo de arroz em dietas de frango de corte.

\section{Material e Métodos}

O experimento foi realizado no Setor de Avicultura do Departamento de Zootecnia da Universidade Federal de Lavras, MG, e no Centro de Energia Nuclear na Agricultura (Cena) da Universidade de São Paulo, Piracicaba, SP, durante o mês de novembro de 1998 .

Foram utilizados 40 frangos de corte machos da linhagem Hubbard, com 27 dias de idade, alojados em gaiolas de metabolismo, distribuídos em cinco tratamentos, quatro repetições e duas aves por parcela experimental. As aves permaneceram alojadas em gaiolas metabólicas durante 14 dias, sendo cinco dias de período pré-experimental para adaptação às gaiolas, e nove dias de período experimental durante o qual realizaram-se as coletas. 
Durante o período de adaptação, todas as aves receberam ração e água à vontade, sendo a ração com níveis nutricionais adequados, visando atender a todas as exigências, segundo National Research Council (1994). No primeiro dia do período experimental, as aves passaram a receber as rações dos respectivos tratamentos, iniciando-se também a coleta total de excretas para determinação do $\mathrm{P}$ inorgânico. A ração fornecida à vontade foi registrada para cálculo do consumo de $\mathrm{P}$ no período experimental. No quinto dia do experimento, todas as aves receberam uma injeção, via peritonial, de $0,5 \mathrm{~mL}$ de $3,500 \mathrm{MBq}$ de ${ }^{32} \mathrm{P}$, como fosfato de sódio $\left(\mathrm{Na}_{3} \mathrm{PHO}_{4}\right)$, livre de carreador.

Amostras de sangue foram coletadas em intervalos de 24 horas, durante cinco dias após a injeção do ${ }^{32} \mathrm{P}$. Durante este período realizou-se também a coleta total das excretas. O sangue, imediatamente após a coleta, foi centrifugado a $3.000 \mathrm{rpm}$ em centrífuga Sorvall, durante 10 minutos, separando-se o plasma para análise. As excretas da primeira etapa do experimento, após coletadas, foram pesadas e armazenadas em congelador. Ao final, foram homogeneizadas por repetição e retirada uma alíquota para determinação do P inorgânico. As excretas da segunda etapa do experimento, após coletadas, foram homogeneizadas retirando-se uma alíquota para análise.

Os tratamentos constituíram-se de cinco dietas: a primeira sem farelo de arroz e sem $\mathrm{P}$ suplementar $(0,386 \% \mathrm{P}$ total) e as outras quatro com $15 \%$ de farelo de arroz, sem $\mathrm{P}$ suplementar $(0,57 \% \mathrm{P}$ total $)$ e com níveis de fitase de 0 , 400, 800 e $1.200 \mathrm{FTU} / \mathrm{kg}$ de dieta.

Uma unidade de fitase (FTU) é definida como sendo a quantidade de enzima necessária para liberar um micromol de $\mathrm{P}$ inorgânico em um minuto, num substrato de sódio-fitato, sob temperatura de $37^{\circ} \mathrm{C}$ e $\mathrm{pH} 5,5$.

As dietas foram formuladas para atender a todas as exigências nutricionais dos frangos de corte, segundo National Research Council (1994), com exceção do P, mantendo-se apenas o $\mathrm{P}$ de origem vegetal.

Os ingredientes utilizados para o preparo das rações, suas respectivas quantidades e o valor nutricional das mesmas estão apresentados na Tabela 1.

A enzima fitase utilizada no experimento foi da marca comercial Natuphos, fornecida pela BASF, e é uma marca registrada da fitase obtida pela fermentação por meio de fungos do grupo Aspergillus niger, que contém conforme o fabricante atividade inicial mínima, de 5.000 FTU/g.

As análises laboratoriais foram realizadas no Laboratório do Centro de Energia Nuclear na Agricultura (Cena) da Universidade de São Paulo, Piracicaba, SP. A análise bromatológica das rações seguiu as recomendações da Association of Official Analytical Chemist (1990).
Nas análises estatísticas, utilizou-se o pacote computacional SAEG (Sistema para Análises Estatísticas e Genéticas). Quando a análise de variância mostrou efeitos significativos para níveis de fitase, realizou-se análise de regressão polinomial. Na comparação dos tratamentos com $15 \%$ de farelo de arroz e níveis de fitase, com o tratamento testemunha sem farelo de arroz, utilizou-se o teste de Dunnett, através do programa computacional SANEST.

\section{Resultados e Discussão}

O consumo de $\mathrm{P}$ não foi afetado significativamente $(\mathrm{P}>0,05)$ pelo nível de fitase das dietas com farelo de arroz (Tabela 2). Entretanto, ele foi significativamente menor no tratamento-testemunha constituído por milho e farelo de soja e com $0,386 \%$ de P total, em relação a todas as dietas com farelo de arroz, com $0,57 \%$ de $\mathrm{P}$ total, independentemente do uso da fitase.

À medida que aumentou o nível de fitase nas rações, houve redução de forma linear $(\mathrm{P}<0,05)$ na excreção total de fósforo. $\mathrm{O}$ aumento da absorção do $P$ com a utilização de fitase, em dietas com a mesma quantidade de $\mathrm{P}$ total, refletiu de maneira significativa na excreção do elemento. Em termos porcentuais, o P total excretado representou $63,6 \%, 50,4 \%, 48,9 \%$ e $49,5 \%$ do total de $\mathrm{P}$ consumido, respectivamente nos níveis de $0,400,800$ e $1.200 \mathrm{FTU} / \mathrm{kg}$.

Tabela 1. Composição e níveis nutricionais das rações experimentais, sem e com $15 \%$ de farelo de arroz integral (FAI) e com quatro níveis de fitase (FTU/kg).

\begin{tabular}{|c|c|c|c|c|c|}
\hline \multirow[t]{3}{*}{ Ingredientes (kg) } & \multirow[t]{3}{*}{$0 \%$ de FAI } & \multicolumn{4}{|c|}{$15 \%$ de FAI } \\
\hline & & 0 & 400 & 800 & 1.200 \\
\hline & & \multicolumn{4}{|c|}{------------ (FTU/kg) ---------- } \\
\hline Milho moído & 64,030 & 49,750 & 49,750 & 49,750 & 49,750 \\
\hline Farelo de soja & 32,410 & 29,880 & 29,880 & 29,880 & 29,880 \\
\hline Farelo de arroz integral & - & 15,000 & 15,000 & 15,000 & 15,000 \\
\hline Calcário calcítico & 2,470 & 2,460 & 2,460 & 2,460 & 2,460 \\
\hline Óleo de soja & 0,333 & 2,150 & 2,150 & 2,150 & 2,150 \\
\hline Premix vitamínico & 0,300 & 0,300 & 0,300 & 0,300 & 0,300 \\
\hline Premix mineral & 0,050 & 0,050 & 0,050 & 0,050 & 0,050 \\
\hline Sal comum & 0,370 & 0,370 & 0,370 & 0,370 & 0,370 \\
\hline Antioxidante (BHT) & 0,015 & 0,015 & 0,015 & 0,015 & 0,015 \\
\hline Fitase (5.000 FTU/g) & - & - & 0,008 & 0,016 & 0,024 \\
\hline Caulim & 0,024 & 0,024 & 0,016 & 0,008 & - \\
\hline Total & 100,00 & 100,00 & 100,00 & 100,00 & 100,00 \\
\hline \multicolumn{6}{|l|}{ Níveis nutricionais } \\
\hline E.M. (kcal/kg) & 2.900 & 2.900 & 2.900 & 2.900 & 2.900 \\
\hline Proteína bruta (\%) & 20,90 & 20,90 & 20,90 & 20,90 & 20,90 \\
\hline Cálcio (\%) & 1,00 & 1,00 & 1,00 & 1,00 & 1,00 \\
\hline P total $(\%)$ & 0,386 & 0,570 & 0,570 & 0,570 & 0,570 \\
\hline P disponível (\%) & 0,145 & 0,165 & 0,165 & 0,165 & 0,165 \\
\hline Fitase (U/kg dieta) & - & - & 400 & 800 & 1.200 \\
\hline
\end{tabular}


A excreção total do $\mathrm{P}$ no tratamento-testemunha foi significativamente inferior $(\mathrm{P}<0,05)$, quando comparada pelo teste de Dunnett aos tratamentos com farelo de arroz, o que está correlacionado à quantidade de $\mathrm{P}$ total na dieta, maior nos tratamentos com farelo de arroz, em relação ao testemunha com milho e farelo de soja. A alta porcentagem de $\mathrm{P}$ do farelo de arroz, aliada à sua baixa disponibilidade, propiciou uma excreção significativamente maior do $\mathrm{P}$ em todos os tratamentos com farelo de arroz.

Considerando-se a excreção de $0,457 \mathrm{~g} / \mathrm{ave} / \mathrm{dia}$, média do tratamento com farelo de arroz e sem fitase, como $100 \%$ de excreção, obteve-se uma redução de $18,1 \%, 20,1 \%$ e $23,4 \%$ com a utilização de 400, 800 e $1.200 \mathrm{FTU} / \mathrm{kg}$. Esses valores são relativamente inferiores aos citados por Simons et al. (1990), Munaro et al. (1996), Conte et al. (1999) e Pizzolante (2000). No presente trabalho, o nível de $\mathrm{P}$ utilizado foi extremamente baixo, inclusive na dieta sem fitase, resultando em excreções menores do que as citadas na maioria dos trabalhos utilizando fitase.

Houve efeito significativo $(\mathrm{P}<0,01)$ dos níveis de fitase (x) sobre o $\mathrm{P}$ absorvido aparente (y), sendo descrito por uma regressão quadrática $\left(\mathrm{Y}=0,263+0,0003 \mathrm{X}-0,0000002 \mathrm{X}^{2}\right)$.

No tratamento-testemunha, o $\mathrm{P}$ absorvido aparente foi de $0,218 \mathrm{~g} /$ ave/dia, não diferindo $(\mathrm{P}>0,05)$ do tratamento com farelo de arroz e sem fitase. Entretanto, foi significativamente menor que os tratamentos com farelo de arroz suplementado com fitase, indicando o efeito desta na absorção do $\mathrm{P}$ da dieta. Em termos porcentuais, a absorção aparente do $\mathrm{P}$ foi de $36,4 \%, 49,6 \%, 51,2 \%$ e $50,5 \%$, com a utilização de $0,400,800$ e $1.200 \mathrm{FTU} / \mathrm{kg}$.
O nível de fitase (x) afetou significativamente $(\mathrm{P}<0,01)$, de forma quadrática $\left(\mathrm{Y}=5,2+0,083 \mathrm{X}-0,00005 \mathrm{X}^{2}\right)$ a excreção endógena de $\mathrm{P}(\mathrm{y})$. No tratamento-testemunha, a excreção de $\mathrm{P}$ foi de $9,5 \mathrm{mg} / \mathrm{ave} / \mathrm{dia}$, não diferindo estatisticamente $(\mathrm{P}>0,05)$ pelo teste de Dunnett, dos níveis de 0 e $400 \mathrm{FTU} / \mathrm{kg}$. O baixo nível de $\mathrm{P}$ das dietas, sem ou com baixo nível de fitase (400 FTU/kg), afetou de maneira importante o metabolismo do $\mathrm{P}$, fazendo com que a ave tivesse uma excreção mínima, na tentativa de manutenção da homeostase do P, possivelmente chegando ao limite mínimo de perda obrigatória do organismo. Entretanto, nos níveis maiores (800 e 1.200 FTU/kg), certamente pelo aumento da disponibilidade para absorção do P contido nos alimentos, a excreção endógena aumentou drasticamente.

Segundo Georgievskii (1982) a excreção de P funciona como importante mecanismo de controle homeostático em monogástricos. Figueirêdo (1998) concluiu que em suínos o trato gastrintestinal desempenha importante papel no controle homeostático do $\mathrm{P}$ do organismo, aumentando ou reduzindo a taxa de absorção, conforme a necessidade do organismo.

No presente trabalho, embora o nível de P total das dietas com FAI tenha sido igual, a presença de níveis crescentes de fitase aumentou a disponibilidade do $\mathrm{P}$ no trato gastrintestinal, aumentando assim o "turnover" do P no organismo, e, conseqüentemente, a sua excreção.

O teor de $\mathrm{P}$ endógeno fecal em aves varia de $10 \%$ a $15 \%$ do $P$ total excretado (Georgievskii, 1982). No presente trabalho, a excreção de P endógeno foi

Tabela 2. Resultados relacionados ao metabolismo do fósforo em frangos de corte, alimentados com dietas sem e com $15 \%$ de farelo de arroz integral (FAI) e com quatro níveis de fitase (FTU/kg).

\begin{tabular}{|c|c|c|c|c|c|c|}
\hline \multirow[t]{3}{*}{ Variáveis } & \multirow[t]{3}{*}{$0 \%$ de FAI } & \multicolumn{4}{|c|}{$15 \%$ de FAI } & \multirow[t]{2}{*}{$\mathrm{CV}(\%)$} \\
\hline & & 0 & 400 & 800 & 1.200 & \\
\hline & & & 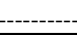 & /kg ----- & --------- & \\
\hline Consumo ração (g/dia) & 130,0 & 128,0 & 132,0 & 133,0 & 126,0 & 5,02 \\
\hline $\mathrm{P}$ consumido (g/dia) & 0,505 & $0,718 *$ & $0,742 *$ & $0,746^{*}$ & $0,707 *$ & 5,02 \\
\hline P total excretado $(\mathrm{g} / \mathrm{dia})^{(1)}$ & 0,287 & $0,457 *$ & $0,374^{*}$ & $0,365 *$ & $0,350 *$ & 8,92 \\
\hline P absorvido aparente $(\mathrm{g} / \mathrm{dia})^{(2)}$ & 0,218 & 0,261 & $0,368 *$ & $0,381 *$ & $0,357 *$ & 6,81 \\
\hline P endógeno fecal (mg/dia) ${ }^{(2)}$ & 9,5 & 12,0 & 14,0 & $76,7 *$ & $62,7 *$ & 16,94 \\
\hline P endógeno fecal (\% do total excretado $)^{(2)}$ & 3,30 & 2,63 & 3,74 & $21,01 *$ & $17,91 *$ & 20,69 \\
\hline P absorvido real (g/dia) ${ }^{(2)}$ & 0,227 & $0,273 *$ & $0,382 *$ & $0,457 *$ & $0,420 *$ & 5,06 \\
\hline Biodisponibilidade do $\mathrm{P}$ da dieta $(\%)^{(2)}$ & 45,02 & $38,06 *$ & $51,54 *$ & $61,31 *$ & $59,54 *$ & 5,76 \\
\hline Biodisponibilidade do $\mathrm{P}$ do $\mathrm{FAI}^{(1)(3)}$ & - & 28,12 & 38,42 & 50,20 & 54,02 & 10,13 \\
\hline P no plasma $(\mathrm{mg} / \mathrm{dl})^{(1)}$ & 3,35 & 3,60 & $5,35^{*}$ & $6,46^{*}$ & $7,32 *$ & 9,25 \\
\hline
\end{tabular}

${ }^{(1)}$ Efeito linear para níveis de fitase $(\mathrm{P}<0,01) .{ }^{(2)}$ Efeito quadrático para níveis de fitase $(\mathrm{P}<0,01) .{ }^{(3)}$ Determinada por fracionamento, excluindo-se o efeito da fitase no $\mathrm{P}$ do milho e farelo de soja. *Diferem da testemunha pelo teste de Dunnett a $5 \%$ de probabilidade. 
de $2,63 \%, 3,74 \%, 21,01 \%$ e $17,91 \%$ do $\mathrm{P}$ total excretado, respectivamente, para os níveis de 0,400 , 800 e $1.200 \mathrm{FTU} / \mathrm{kg}$. Assim, pode-se inferir, através destes resultados, que a excreção endógena representa uma resposta fisiológica do animal, e no presente trabalho, os níveis maiores, de 800 e 1.200 FTU/kg, permitiram à ave atingir um status adequado de P no organismo, já que a excreção foi ligeiramente superior ao considerado normal por Georgievskii (1982)

O efeito da enzima fitase (x) sobre a absorção verdadeira de $\mathrm{P}$ (y) foi descrito por uma regressão quadrática $\left(\mathrm{Y}=0,269+0,000415 \mathrm{X}-0,00000024 \mathrm{X}^{2}\right) \mathrm{e}$, por meio desta, determinou-se que o ponto de máxima absorção foi de $0,448 \mathrm{~g} /$ ave/dia quando foram utilizados $864 \mathrm{FTU} / \mathrm{kg}$ de dieta.

Com os resultados de absorção verdadeira do $\mathrm{P}$ obteve-se a disponibilidade real do $\mathrm{P}$ das dietas, sendo de $38,0 \%, 51,5 \%, 61,3 \%$ e $59,5 \%$, respectivamente, para os níveis de 0, 400, 800 e $1.200 \mathrm{FTU} / \mathrm{kg}$.

No tratamento testemunha a biodisponibilidade do $\mathrm{P}$ foi de $45,02 \%$, sendo significativamente maior $(\mathrm{P}<0,05)$ do que o tratamento com farelo de arroz sem fitase. Esse valor está de acordo com Nelson (1980), que verificou uma disponibilidade variando de $19 \%$ a 44\%, quando as dietas utilizadas continham apenas $\mathrm{P}$ de origem vegetal. Segundo Bellaver et al. (1983), pode ocorrer uma variação de $18 \%$ a $60 \%$ na disponibilidade do $\mathrm{P}$ de dietas contendo milho e farelo de soja, e esta variação se deve principalmente ao tipo de animal, ao tipo de alimento e à técnica utilizada.

Por meio da equação de regressão, que descreveu o efeito dos níveis de fitase $(\mathrm{x})$ sobre a biodisponibilidade do $\mathrm{P}(\mathrm{y}) \operatorname{das} \operatorname{dietas}(\mathrm{Y}=37,66+$ $\left.0,04715 \mathrm{X}-0,000024 \mathrm{X}^{2}\right)$, obteve-se o ponto de máxima biodisponibilidade, que foi de $60,8 \%$, quando da utilização de 982 FTU/kg.

Em termos porcentuais, a utilização da fitase propiciou um aumento da biodisponibilidade do $\mathrm{P}$ das dietas de $33,5 \%, 58,8 \%$ e $56,4 \%$ quando utilizaram-se, respectivamente, 400, 800 e $1.200 \mathrm{FTU} / \mathrm{kg}$, em relação à dieta com farelo de arroz e sem fitase.

Com base nos resultados de biodisponibilidade do $\mathrm{P}$ da dieta-controle (testemunha), quando utilizou-se apenas o milho e o farelo de soja como fontes de $\mathrm{P}$ e nos tratamentos com $15 \%$ de farelo de arroz, calculou-se, por meio do fracionamento do $\mathrm{P}$ consumido e do P absorvido verdadeiro, a biodisponibilidade do $\mathrm{P}$ do farelo de arroz a qual foi de $28,1 \%$, aumentando para $38,4 \%, 50,2 \%$ e $54,0 \%$ com a utilização de 400, 800 e 1.200 FTU/kg, respectivamente. O efeito da fitase $(\mathrm{x})$ sobre a biodisponibilidade do $\mathrm{P}(\mathrm{y})$ do farelo de arroz foi significativo $(\mathrm{P}<0,01)$ e foi descrito por uma equação linear $(Y=29,272+0,0223 X)$.

$\mathrm{O}$ valor de $28,1 \%$ na biodisponibilidade do $\mathrm{P}$ do farelo de arroz é bastante superior ao da maioria dos dados citados em tabelas de composição de alimentos, tais como 14,3\% (Rostagno et al., 1994) e 14,6\% (National Research Council, 1994).

Valores altos de biodisponibilidade do $\mathrm{P}$ do farelo de arroz podem ser justificados pela utilização de níveis extremamente baixos de $\mathrm{P}$ disponível na dieta, o que leva o animal a alterar o seu metabolismo, aumentando a absorção e reduzindo a excreção endógena.

$\mathrm{O}$ efeito da fitase sobre a biodisponibilidade do $\mathrm{P}$ do farelo de arroz é coerente com os dados de literatura. No presente trabalho, a ação da fitase representou um acréscimo de $36,2 \%, 78,6 \%$ e $92 \%$ na biodisponibilidade do $\mathrm{P}$, quando foram utilizadas 400 , 800 e $1.200 \mathrm{FTU} / \mathrm{kg}$.

$\mathrm{O}$ nível de fitase $(\mathrm{x})$ afetou significativamente $(\mathrm{P}<0,01)$ o teor de $\mathrm{P}(\mathrm{y})$ no plasma, sendo o efeito descrito pela equação $\mathrm{Y}=3,844+0,00307 \mathrm{X}$.

Os resultados obtidos neste trabalho são similares aos encontrados por Munaro (1993) utilizando dietas com farelo de arroz e níveis crescentes de fitase. $\mathrm{O}$ autor obteve aumento linear significativo do $\mathrm{P}$ do plasma com o aumento do nível de fitase na ração. Perney et al. (1993) e Pizzolante (2000) também obtiveram aumento linear do $\mathrm{P}$ plasmático com suplementação de níveis crescentes de fitase em dietas de origem vegetal.

Estes resultados indicam um maior "turnover" de P no organismo, fato que pode ser comprovado pelo aumento da absorção e da excreção endógena de $\mathrm{P}$, quando se utilizam níveis crescentes de fitase na ração.

\section{Conclusões}

1. A utilização da enzima fitase aumenta a absorção verdadeira de $\mathrm{P}$, o teor de $\mathrm{P}$ no plasma, e a sua excreção endógena.

2. A utilização da enzima fitase melhora a 
biodisponibilidade do P nas dietas com $15 \%$ de farelo de arroz; com 982 FTU $/ \mathrm{kg}$, obtém-se máxima biodisponibilidade de fósforo.

3. A biodisponibilidade do $\mathrm{P}$ do farelo de arroz aumenta de forma linear com doses crescentes de fitase.

\section{Referências}

ASSOCIATION OF OFFICIAL ANALYTICAL CHEMIST (Gaithersburg, Estados Unidos). Official methods of analysis. 15. ed. Arlington, 1990. $1078 \mathrm{p}$.

BELLAVER, C.; GOMES, P. C.; SANTOS, D. L. Absorção e disponibilidade de fósforo para suínos, baseada na diluição de radioisótopos $\left({ }^{32} \mathrm{P}\right)$. Pesquisa Agropecuária Brasileira, Brasília, v. 9, n. 18, p. 1053-1057, set. 1983.

BORGES, F. M. de O. Utilização de enzimas em dietas avícolas. Cadernos Técnicos da Escola de Veterinária da UFMG, Belo Horizonte, n. 20, p. 5-30, jun. 1997.

CONTE, A. J.; TEIXEIRA, A. S.; FIGUEIRÊDO, A. V.; SOUZA, B. B. Efeito da fitase na disponibilidade de fósforo e no desempenho de frangos de corte alimentados com dietas contendo farelo de arroz integral. In: REUNIÃO ANUAL DA SOCIEDADE BRASILEIRA DE ZOOTECNIA, 36., 1999, Porto Alegre. Anais... Porto Alegre: Gnosis, 1999. 1 CD-ROM.

FIGUEIRÊDO, A. V. Disponibilidade biológica do fósforo de cinco fosfatos, determinada em suínos em crescimento, através da técnica de diluição isotópica. 1998. 103 f. Tese (Doutorado) - Centro de Energia Nuclear na Agricultura, Universidade de São Paulo, Piracicaba. 1998.

GEORGIEVSKII, V. I. The physiological role of macroelements. In: GEORGIEVSKII, V. I.; ANNENKOW, B. N.; SAMOKHIN, V. T. Mineral nutrition of animals. London: Butterworths, 1982. p. 91-170.

KLEIBER, M.; SMITS, A. H.; RASLTON, N. P.; BLACK, A. L. Radiophosphorus $\left({ }^{32} \mathrm{P}\right)$ as tracer for measuring endogenous phosphorus in cow's feces. Journal of Nutrition, Bethesda, v. 45, n. 2, p. 252-263, 1951.

MUNARO, F. A. Efeito da fitase na biodisponibilidade do fósforo do farelo de arroz desengordurado em rações para frangos de corte. 1993. $174 \mathrm{f}$. Tese (Mestrado) - Universidade Federal do Rio Grande do Sul, Porto Alegre. 1993.

MUNARO, F. A.; LÓPEZ, J.; LÓPEZ, S. E.; RÜTZ, F. Efeito da fitase na biodisponibilidade do fósforo em rações com farelo de arroz desengordurado para frangos de corte. Revista da Sociedade Brasileira de Zootecnia, Viçosa, v. 25 , n. 5, p. 932-943, 1996.

NATIONAL RESEARCH COUNCIL (Washington, Estados Unidos). Nutrient requirements of poultry. 9. ed. Washington: National Academy, 1994. 155 p.

NELSON, T. S. Phosphorus availability in plant origin feedstuffs or poultry and swine. In: ANNUAL INTERNATIONAL MINERALS CONFERENCE, 3 ., 1980, Orlando. Proceedings... Orlando: International Minerals \& Chemical Corporation, 1980. p. 59-84.

NELSON, T. S. The utilization of phytate phosphorus by poultry: a review. Poultry Science, Champaign, v. 46, n. 4 , p. 862-871, Apr. 1967

NELSON, T. S.; SHIEH, T. R.; WODZINSKI, R. J.; WARE, J. H. The availability of phytate phosphorus in soybean meal before and after treatment with a mold phytase. Poultry Science, Champaign, v. 47, p. 1842$1848,1968$.

PERNEY, M.; CANTOR, A. H.; STRAW, M. L.; HERKELMANN, K. L. The effect of dietary on growth performance and phosphorus utilization of broiler chicks. Poultry Science, Campaign, v. 72, n. 11, p. 2106-2114, Nov. 1993.

PIZZOLANTE, C. C. Estabilidade da fitase e sua utilização na alimentação de frangos de corte. $2000.117 \mathrm{f}$. Tese (Doutorado) - Universidade Federal de Lavras, Lavras. 2000

ROSTAGNO, H. S.; SILVA, D. J.; COSTA, P. M. A.; FONSECA, J. B.; SOARES, P. R.; PEREIRA, J. A. A.; SILVA, M. A. Composição de alimentos e exigências nutricionais de aves e suínos: tabelas brasileiras. Viçosa, MG: UFV, 1994. 59 p.

SIMONS, P. C.; VERSTEEGH, H. A.; JONGBLOED, A. W.; KEMME, P. A.; SLUMP, P.; BOS, K. D.; WOLTERS, M. G.; BEUDEKER, R. F.; VERSCHOOR, G. J. Improvement of phosphorus availability by microbial phytase in broilers and pigs. British Journal of Nutrition, Wallingford, v. 64, p. 525-540, Sept. 1990.

VITTI, D. M. S. S.; ABDALLA, A. L.; SILVA FILHO, J. C. da. Avaliação da disponibilidade biológica do fósforo do fosfato bicálcico e de fosfatos de rocha para ovinos com uso de radiofósforo $\left({ }^{32} \mathrm{P}\right)$ como traçador. Pesquisa Agropecuária Brasileira, Brasília, v. 26, n. 8, p. 11131118, ago. 1991. 